\title{
The Helium Field Effect Transistor (I): Storing Surface State Electrons on Helium Films
}

\author{
M. Ashari • D.G. Rees • K. Kono • E. Scheer • \\ P. Leiderer
}

\begin{abstract}
We present investigations of surface state electrons on liquid helium film in confine geometry, using a suitable substrate structure microfabricated on a silicon wafer, similar to a Field Effect Transistor (FET). The sample has a source and drain region, separated by a gate structure, which consists of two gold electrodes with a narrow gap (channel) through which the transport of the surface state electrons takes place. The sample is illuminated to provide a sufficien number of free carriers in the silicon substrate, such that a well-define potential distribution is achieved. The eventual goal of these experiments is to study the electron transport through a narrow channel in the various states of the phase diagram of the 2D electron system. In the present work we focus on storing the electrons in the source area of the FET, and investigate the spatial distribution of these electrons. It is shown that under the influ ence of a potential gradient in the silicon substrate the electrons accumulate in front of the potential barrier of the gate. The electron distribution, governed by Coulomb repulsion and by the substrate potential, is determined experimentally. The result is found to be in good agreement with a parallel-plate capacitor model of the system, developed with the aid of a finit element calculation of the surface potential profil of the device.
\end{abstract}

Keywords Helium fiel effect transistor · Surface electron · Helium fil · Electron storage

\footnotetext{
M. Ashari - E. Scheer · P. Leiderer $(\bowtie)$

Department of Physics, University of Konstanz, Konstanz 78457, Germany

e-mail: paul.leiderer@uni-konstanz.de

D.G. Rees - K. Kono

RIKEN, Hirosawa 2-1, Wako, Saitama, Tokyo, 351-0198, Japan
}

Konstanzer Online-Publikations-System (KOPS) URL: http://nbn-resolving.de/urn:nbn:de:bsz:352-194460 


\section{Introduction}

Electrons on the surface of liquid helium have been studied quite extensively as an example of a particularly well-define and clean Coulomb system [1, 2]. In most of the investigations the properties of the quasi-infinit 2-dimensional system of electrons on bulk helium have been considered and many prominent effects characteristic of charged systems, like magnetotransport phenomena and Wigner crystallization, have been observed [3, 4]. More recently also electrons in confined geometry, e.g. in channels fille with liquid helium due to capillarity, have revealed intriguing effects in the charge transport of these systems [5, 6]. Lately, due to their high mobility and long coherence time, 2D electrons on helium have been discussed as a system for testing concepts of quantum computing [7, 8]. In contrast to the well-known 2D electrons at semiconductor interfaces, electrons on liquid helium in nearly all the measurements carried out so far were not in the high density range of the degenerate Fermi gas, but in the classical flui or crystal regime, since the charge density is limited to values $n<n_{\mathrm{c}}=2 \times 10^{9} \mathrm{~cm}^{-2}$ on bulk ${ }^{4} \mathrm{He}$ (and even lower on ${ }^{3} \mathrm{He}$ ) because of an electrohydrodynamic instability [9]. For surfaces in confine geometry the limit in the electron density is somewhat increased due to the stabilizing action of surface tension, but considerably higher densities on the order of $10^{11} \mathrm{~cm}^{-2}$, which are required to reach the degenerate Fermi regime, are only accessible on thin helium films. Electrons on helium film thus lend themselves not only for tracing out the regime of the classical Coulomb flui and its transition to the Wigner crystal, but also the quantum melting of the Wigner crystal towards the degenerate Fermi gas [10].

A benchmark experiment for revealing the nature of electron transport is given by studying the $\mathrm{fl} \mathrm{w}$ of electrons through a one-dimensional channel, the width of which can be controlled independently. For degenerate electron gases in semiconductor heterojunctions, these studies led to the discovery of conductance quantization in quantum point contacts [11-13]. This line of investigation is also of particular interest for electrons on liquid ${ }^{4} \mathrm{He}$ film in which the various regimes of the $2 \mathrm{D}$ phase diagram may be addressed. A crucial information for obtaining a quantitative understanding of the transport is the knowledge of the density and the spatial distribution of the electrons on the surface. In this experiment we use a source-gate-drain config uration similar to a Metal-Oxide-Semiconductor Field Effect Transistor (MOSFET) supported by a silicon substrate [14]. In the present paper we focus on the spatial distribution of the electrons, as they are stored in the source area of the He FET, under the influenc of a potential gradient in the silicon substrate.

\section{Experimental Set-up and Method}

The sample used in this work was a rectangular piece of a $0.3 \mathrm{~mm}$ thick silicon wafer $22 \times 18 \mathrm{~mm}$ in size (see Fig. 1). The Si was covered by a $500 \mathrm{~nm}$ thick oxide layer, and several gold electrodes on top, prepared by photolithography, provided the structures for the FET. The source (S) and drain (D) areas were separated by a split gate (G) with a $10 \mu \mathrm{m}$ wide and $100 \mu \mathrm{m}$ long channel, and a guard electrode surrounded the S-G-D structure to confin the surface state electrons (SSE) to the FET area. The Si 
Fig. 1 (Color online)

Photograph of the He FET. The size of the sample is

$22 \times 18 \mathrm{~mm}$. It is covered by an insulating oxide layer with a thickness of $500 \mathrm{~nm}$. The evaporated gold electrodes are $100 \mathrm{~nm}$ thick. The right hand side shows a scanning electron microscope image of the gate channel, which is $100 \mu \mathrm{m}$ long and $10 \mu \mathrm{m}$ wide

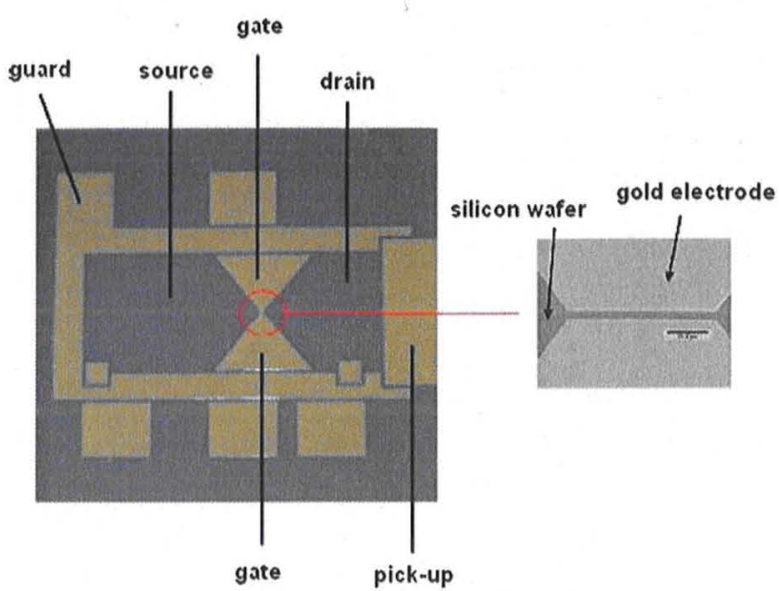

substrate had ohmic contacts at the S and D edge of the wafer. The voltages applied to the various contacts were typically in the range of a few volt.

In operation, the sample was cooled to a temperature of $1.3 \mathrm{~K}$, with the sample surface slightly above the bulk liquid helium level. The FET structure was therefore covered with a thin superflui $\mathrm{He}$ fil with a thickness $d_{\mathrm{He}}$ depending on the height of the surface above the bulk liquid. In the experiments reported here this height was $2.5 \mathrm{~mm}$, corresponding to a He fil thickness $d_{\mathrm{He}} \approx 50 \mathrm{~nm}[15]$.

In order to charge the source $\mathrm{S}$, we used a small filament mounted several $\mathrm{mm}$ above the source area, which was heated with short $(25 \mathrm{~ms})$ current pulses. Thermionic emission then gave rise to about $10^{7}$ electrons per pulse. They were pulled towards the source and trapped in the image potential well on the fil surface, where they redistributed according to the potentials applied to the FET electrodes. Electrons reaching the drain area were collected by a pick-up electrode and registered with a sensitive electrometer.

\section{Results and Discussion}

\subsection{Potential Distribution of the Uncharged FET Structure}

Since the Si used here as a substrate was undoped, its resistance diverges rapidly at low temperature, which leads to an ill-define potential distribution in the presence of surface state electrons. In order to increase the conductivity we illuminated the sample continuously during each experimental run using an external light source, thus generating a sufficien number of electrons and holes in the Si that the resistance between the $\mathrm{S}$ and $\mathrm{D}$ contact dropped to $\approx 1 \mathrm{M} \Omega$. The electrostatic potential measured under these conditions at four positions between $\mathrm{S}$ and $\mathrm{D}$ by additional contacts at the back of the Si wafer is shown in Fig. 2. In the whole voltage range up to $10 \mathrm{~V}$ the profil is nearly linear and so the electric fiel is approximately uniform within the $\mathrm{Si}$ wafer. This is important for analyzing the SSE distribution in the source, as will be shown further below. 
Fig. 2 (Color online) Potential distribution in the Si substrate for different source-drain voltages, as shown in the legend. The data were taken at $1.3 \mathrm{~K}$ and with illumination. S (D) denotes the position of the source (drain) electrode

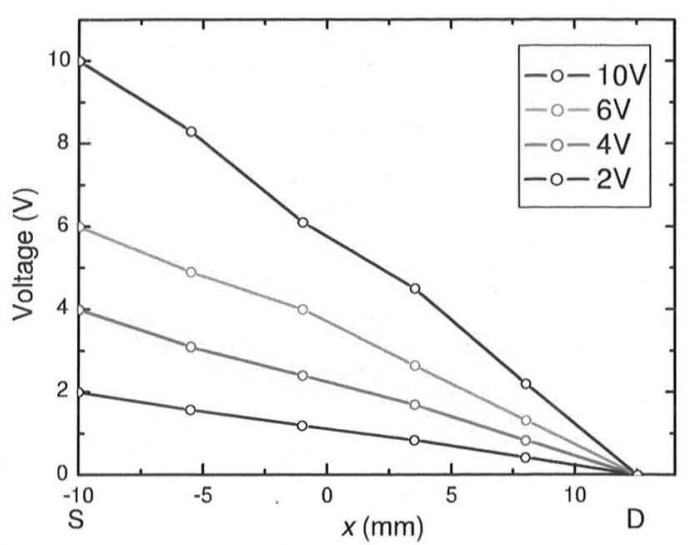

In addition to these pointwise measurements we used a computer simulation to determine the potential energy landscape in the FET structure, including the gate and guard electrodes. We defin the electrostatic potential energy on the uncharged helium surface as $\Phi_{\text {sur }}(x, y)=-e V(x, y)$, where $e$ is the magnitude of the electron charge ( $-e$ is the electron charge) and $V(x, y)$ is the voltage on the helium surface with respect to ground, at the location $x, y$. Since we have two separated Coulomb systems here, on the one hand the surface state electrons, on the other hand the charge carriers in the substrate, we also defin the electrostatic potential energy within the substrate, $\Phi_{\text {sub }}$. The channel is oriented along the $x$ direction $(x=0$ to $x=0.1 \mathrm{~mm}$ ) and is located around $y=0$ (from $y=-5 \mu \mathrm{m}$ to $y=5 \mu \mathrm{m}$ ). As the channel lies approximately midway between the source and drain, the value of $\Phi_{\text {sub }}$ at the center of the channel can be estimated as $\Phi_{\text {sub }}^{0}=-e V_{0}$ where $V_{0} \approx\left(V_{\mathrm{d}}-V_{\mathrm{S}}\right) / 2$. We defin the potential on the helium surface at the center of the channel as $\Phi_{\text {sur }}^{0}$

Figure 3a shows a schematic representation of $\Phi_{\text {sur }}$ across the sample surface. The voltages applied to source and drain are chosen here as $V_{\mathrm{s}}=+1 \mathrm{~V}$ and $V_{\mathrm{d}}=+2 \mathrm{~V}$, and the guard voltage is $V_{\text {guard }}=-1.5 \mathrm{~V}$. By applying a negative voltage to the two gate electrodes (with respect to the voltage of the Si substrate at the gate position) a potential barrier is generated between source and drain. In Fig. 3b, which is a schematic plot of $\Phi_{\text {sur }}(x)$ along the center of the sample $(y=0)$, the potential barrier shows up as a sharp spike at the location of the gate channel reaching from $x=0$ to $0.1 \mathrm{~mm}$. We defin the height of the potential barrier as $\Phi_{\text {bar }}=\Phi_{\text {sur }}^{0}-\Phi_{\text {sub }}^{0}=$ $-k e\left(V_{\mathrm{g}}-V_{0}\right)$, where $k$ is a constant of proportionality. Figure 3c, which shows results of the simulation, gives a blow-up of the potential in the central part of the gate channel for $V_{\mathrm{g}}=-10 \mathrm{~V}, V_{0}=1.5 \mathrm{~V}$. Figure $3 \mathrm{~d}$ shows a profil of the potential across the channel for various gate voltages, for $V_{0}=0 \mathrm{~V}$. Here, because $V_{0}=0 \mathrm{~V}$, the potential at the center of the channel gives the barrier height directly. As expected, the potential at the center of the channel is distinctly smaller than the potential of the gate itself. The constant $k$ depends on the thickness $d_{\mathrm{He}}$ of the helium film as is obvious from Fig. 3e, in which we plot the barrier height for different gate voltages and $d_{\mathrm{He}}$, for $V_{0}=0 \mathrm{~V}$. For $d_{\mathrm{He}}=50 \mathrm{~nm}$-the situation of our measurements-the simulations yield a value of $k=0.024$. 
a)

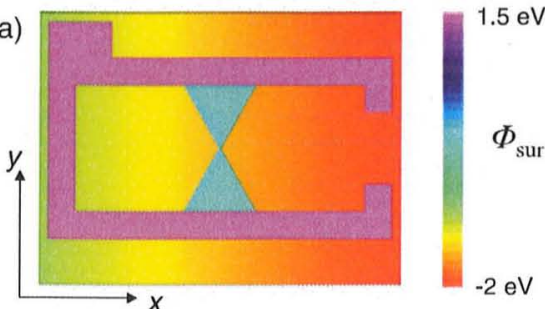

c)

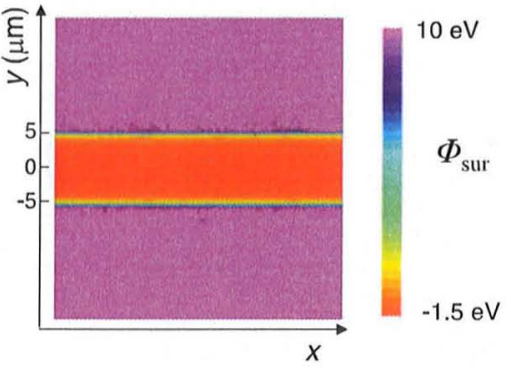

b)

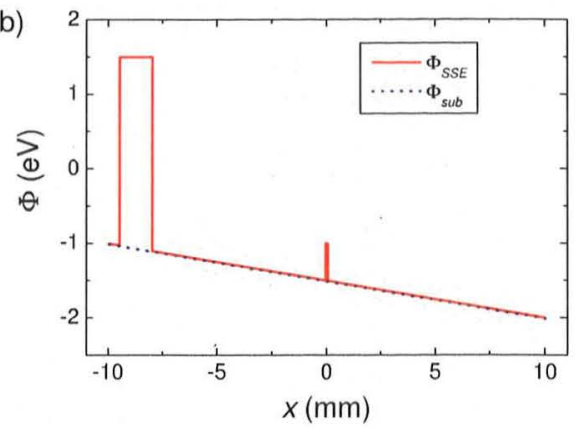

d)

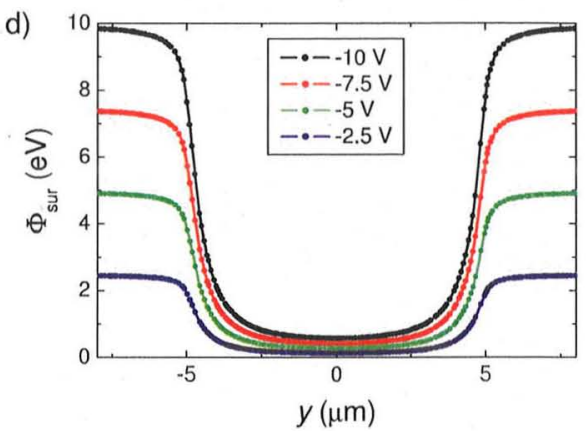

e)

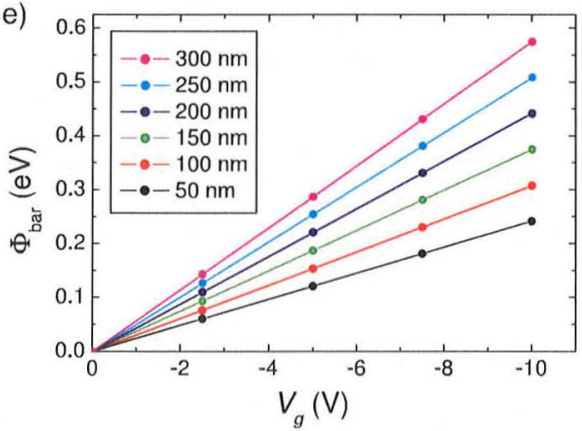

Fig. 3 (Color online) (a) Schematic of the potential energy landscape $\Phi_{\text {sur }}(x, y)$ on the He f Im covering the FET structure. Here $V_{\mathrm{g}}=0 \mathrm{~V}, V_{\text {guard }}=-1.5 \mathrm{~V}, V_{\mathrm{s}}=1 \mathrm{~V}$ and $V_{\mathrm{d}}=2 \mathrm{~V}$. (b) Schematic profil of the potential energy along the center of the sample between the source and drain, for the same bias conditions. The narrow spike at $x=0$ corresponds to the position of the 1D channel. (c) Potential energy landscape for the gate channel obtained from the simulation for $V_{\mathrm{g}}=-10 \mathrm{~V}, V_{0}=1.5 \mathrm{~V}$ and $d_{\mathrm{He}}=300 \mathrm{~nm}$. (d) Potential profil across the channel obtained from the simulation for various values of $V_{\mathrm{g}}$, as shown in the legend. Here $V_{0}=0 \mathrm{~V}$ and $d_{\mathrm{He}}=300 \mathrm{~nm}$. (e) The dependence of barrier height on $V_{\mathrm{g}}$ according to the simulation, at various helium fil thicknesses, as shown in the legend. Here $V_{0}=0 \mathrm{~V}$. The slope of the line gives the coupling constant $k$, which for $d_{\mathrm{He}}=50 \mathrm{~nm}$ is $k=0.024$

\subsection{Charging the Source}

We now consider the charging procedure of the source and assume for that purpose that the gate is negatively biased so that it provides a potential barrier, as schematically depicted in Fig. 4. As the filamen is activated, the electrons supplied by the 
a)

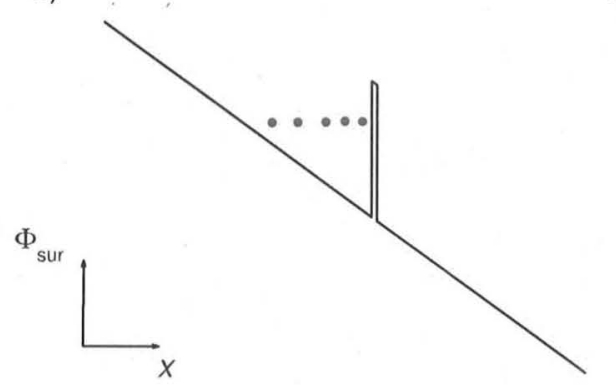

b)

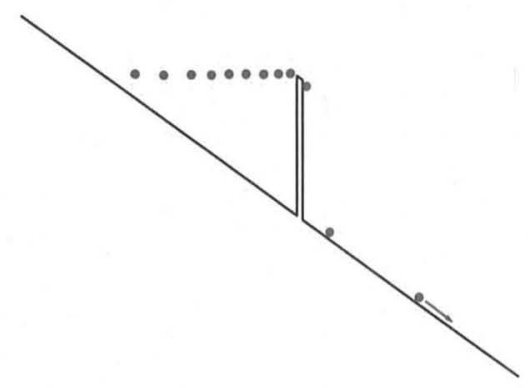

Fig. 4 (Color online) Schematic potential profil along the $x$-axis of the sample for (a) charging the source and (b) electron 'spill-over' when the chemical potential of the electron system is equal to the barrier potential

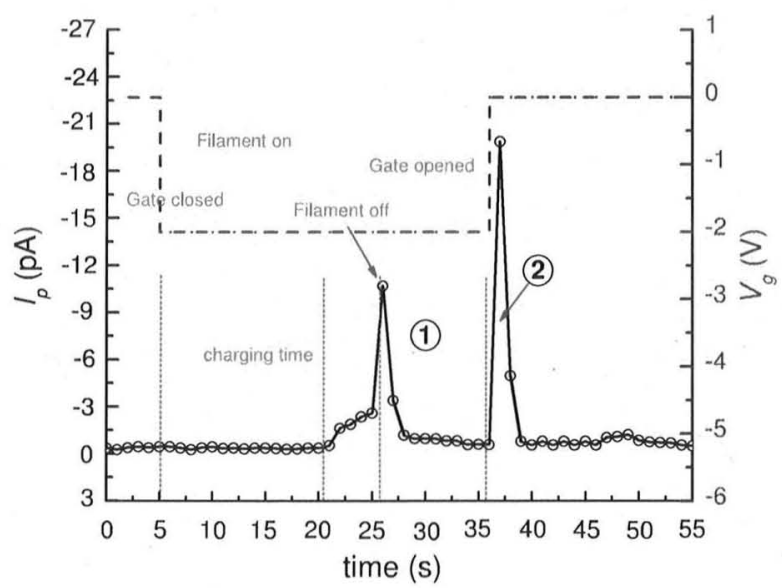

Fig. 5 (Color online) Procedure for fillin the source and determining the number of stored electrons. Here $V_{\text {guard }}=-1.5 \mathrm{~V}, V_{\mathrm{s}}=1 \mathrm{~V}$ and $V_{\mathrm{d}}=2 \mathrm{~V}$. The pick-up current $I_{\mathrm{p}}$ shows the spilling over of electrons when the source is full. At the beginning, the source is empty. Then the following steps are taken: First, the gate is closed by switching $V_{\mathrm{g}}$ from 0 to $-2 \mathrm{~V}$. Then the filamen is pulsed periodically at a rate of 4 pulses/s, and charging of the source begins. $I_{\mathrm{p}}$ remains zero because of the closed gate. After a fillin time of about $10 \mathrm{~s}$ an onset of $I_{\mathrm{p}}$ is observed, indicating that the source is fille to its maximum capacity and electrons begin to spill over the barrier (peak nr. 1). The filamen is then switched off, and $I_{\mathrm{p}}$ drops back to zero. After a certain waiting time - in this case 8 seconds - the gate is opened and the charges stored in the source area pass the channel and are collected by the pick-up electrode. This gives rise to peak nr. 2 in the pick-up current, which integrated in time represents the number of stored electrons $N_{\mathrm{s}}$. In the example shown here $N_{\mathrm{s}}$ is $1.5 \times 10^{8}$

firs charging pulse will move under the influenc of the substrate potential towards the entrance of the gate channel, where they are blocked by the gate barrier. As more and more charges are added, the electron pool increases in size and the chemical potential of the electrons rises due to their Coulomb interaction, until eventually it is high enough that electrons start spilling over the barrier, pass through the channel and reach the drain (Fig. 4b). An example for this process is shown in Fig. 5, where the pick-up current $I_{\mathrm{p}}$ is plotted as a function of time. 

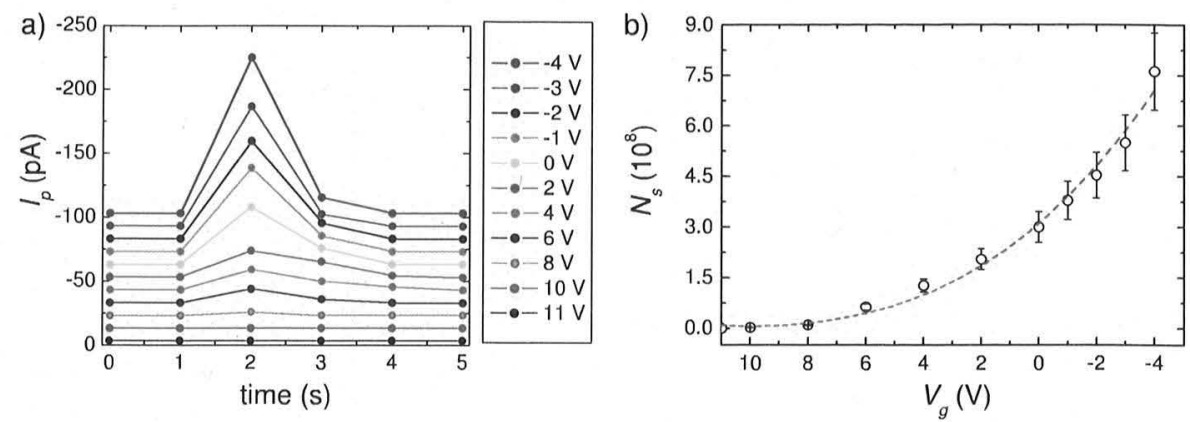

Fig. 6 (Color online) (a) Pick-up current as a function of time for different gate voltages, as shown in the legend, and $V_{\text {guard }}=-7 \mathrm{~V}, V_{\mathrm{s}}=10 \mathrm{~V}, V_{\mathrm{d}}=12 \mathrm{~V}$. (b) Number of stored electrons, $N_{\mathrm{s}}$, as a function of gate voltage. The dashed curve is a fi to the model described in Sect. 3.4

\subsection{Storage Capacity of the Source}

We have carried out the procedure described above for different values of $V_{\mathrm{g}}$, fillin in each case the source until spill-over of the electrons took place, and then determined the number of stored electrons $N_{\mathrm{s}}$. The result is shown in Fig. 6 for $V_{\text {guard }}=-7 \mathrm{~V}$, $V_{\mathrm{s}}=10 \mathrm{~V}, V_{\mathrm{d}}=12 \mathrm{~V}$ and $V_{0}=11 \mathrm{~V}$. As $V_{\mathrm{g}}$ and hence the potential barrier in the channel is increased, the number of stored electrons also increases, as expected. However, somewhat unexpectedly - at least at firs glance- the relation between $V_{\mathrm{g}}$ and $N_{\mathrm{S}}$ is distinctly nonlinear.

In order to make sure that this nonlinear behavior is not an experimental artefact, we have repeated the measurement of the number of stored electrons using a slightly modifie method as a consistency check (see Fig. 7). As in the procedure shown in Fig. 5, the source was fille at a certain $V_{\mathrm{g}}$ until electron spill-over was observed. Then, however, the gate was not opened completely in one large, but rather in several small steps, and the charge reaching the pick-up after each step was registered. From the current peaks, the total number of electrons stored in the source for each value of $V_{\mathrm{g}}$ can be determined, with a result similar to Fig. 6. (The values of $V_{\text {guard }}, V_{\mathrm{s}}$ and $V_{\mathrm{d}}$ were identical to those in Fig. 6.)

\subsection{Model for the Electron Distribution in the Source}

The nonlinear storage capacity for surface state electrons, as it is presented in Figs. 6 and 7, can be explained by an inhomogeneous electron density $n(x)$ in the source area. This inhomogeneity, which results from the potential gradient in the Si substrate, is schematically sketched in Fig. 8. Qualitatively, on the basis of Fig. 4, one expects the highest electron density at the entrance of the gate channel. More quantitatively, we consider the system of surface state electrons and the Si substrate as two plates of a parallel capacitor, whose charge density will vary because the potential difference between the two plates depends on the $x$-coordinate. Whereas the chemical potential of the surface state electron system is constant throughout the charged area, ${ }^{1}$ the

\footnotetext{
${ }^{1}$ In the absence of a transport current the charges arrange in such a way that this condition is fulfilled
} 
a)

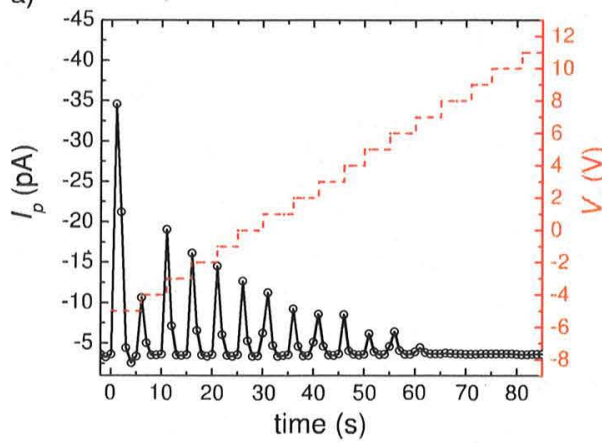

b)

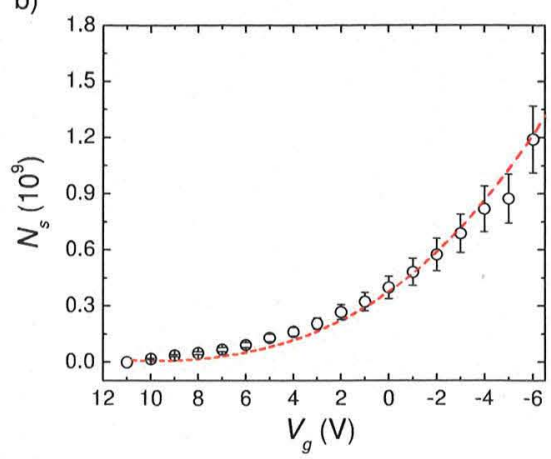

Fig. 7 (Color online) (a) Pick-up current as a function of time for a stepwise reduction of the gate voltage. (b) Number of stored electrons, $N_{\mathrm{s}}$, as a function of gate voltage. The dashed curve is a fi to the model described in Sect. 3.4

Fig. 8 (Color online) Schematic distribution of the surface state electrons in the source region when a potential gradient between the $\mathrm{S}$ and $\mathrm{D}$ contacts exists

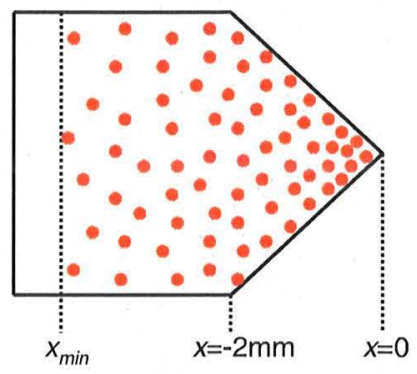

potential of the Si substrate varies along the $x$-direction according to Fig. 2. From this model we expect a linear drop of $n(x)$, from a maximum density $n(0)$ at the channel entrance to $n=0$ at a certain value of $x$ (here called $x_{\min }$ ) which depends on the total charge, the potential gradient between source and drain and the height of the potential barrier $\Phi_{\text {bar }}$. On the basis of the capacitor model, $n(x)$ is given by

$$
n(x)=\frac{\varepsilon_{0} \varepsilon_{1} \varepsilon_{2} V(x)}{\left(\varepsilon_{1} d_{\mathrm{He}}+\varepsilon_{2} d_{\mathrm{S}}\right) e}=\frac{\varepsilon_{0} \varepsilon_{1} \varepsilon_{2} c\left(x-x_{\mathrm{min}}\right)}{\left(\varepsilon_{1} d_{\mathrm{He}}+\varepsilon_{2} d_{\mathrm{S}}\right) e} .
$$

Here $\varepsilon_{0}, \varepsilon_{1}, \varepsilon_{2}, d_{\mathrm{S}}, d_{\mathrm{He}}$ and $c$ are the vacuum permittivity, relative silicon oxide permittivity (3.9), relative helium permittivity (1.055), silicon oxide thickness $(500 \mathrm{~nm})$, helium fil thickness $(50 \mathrm{~nm})$ and the electric fiel $\left(2 \mathrm{~V} / 2.2 \mathrm{~cm}=0.91 \mathrm{~V} \mathrm{~cm}^{-1}\right)$ within the substrate, respectively. $V(x)$ is the voltage which corresponds to the depth of the potential well in which the electrons are stored. $V(x)$ is zero for $x=x_{\min }$ and reaches a maximum of $-\Phi_{\mathrm{bar}} / e$ at the channel entrance, for the case in which the source is charged until electrons spill over the barrier (i.e. the electrostatic energy of the electrons due to Coulomb repulsion is equal to $\Phi_{\text {bar }}$, as in Fig. 4b). The total number of stored electrons is calculated by integrating over the electron distribution in the source:

$$
N_{\mathrm{s}}=\int_{x_{\min }}^{0} n(x) W(x) d x
$$


where $W(x)$ is the width of the source at the position $x$. For $-2 \mathrm{~mm} \leq x_{\min } \leq 0 \mathrm{~mm}$, the source area is triangular and $W(x)=b x$ where $b$ is a constant. For our sample geometry $b=6.5 \mathrm{~mm} /-2 \mathrm{~mm}=-3.25$. For $x<-2 \mathrm{~mm}$, the source is rectangular with width $6.5 \mathrm{~mm}$. For $-2 \mathrm{~mm} \leq x_{\min } \leq 0 \mathrm{~mm}$, using (1) we obtain

$$
N_{\mathrm{S}}=\frac{\varepsilon_{0} \varepsilon_{1} \varepsilon_{2} c b}{\left(\varepsilon_{1} d_{\mathrm{He}}+\varepsilon_{2} d_{\mathrm{S}}\right) e} \int_{x_{\min }}^{0} x\left(x-x_{\mathrm{min}}\right) d x,
$$

and finall

$$
N_{\mathrm{s}}=\frac{\varepsilon_{0} \varepsilon_{1} \varepsilon_{2} b c}{6\left(\varepsilon_{1} d_{\mathrm{He}}+\varepsilon_{2} d_{\mathrm{S}}\right) e} x_{\min }^{3} .
$$

As stated above, $V(x)$ ranges from 0 at $x_{\min }$ to $-\Phi_{\text {bar }} / e$ at the channel entrance, with the voltage gradient $c$. Therefore, since $x_{\min }=k\left(V_{\mathrm{g}}-V_{0}\right) / c$, we obtain

$$
N_{\mathrm{S}}=\frac{\varepsilon_{0} \varepsilon_{1} \varepsilon_{2} b k^{3}}{6\left(\varepsilon_{1} d_{\mathrm{He}}+\varepsilon_{2} d_{\mathrm{S}}\right) c^{2} e}\left(V_{\mathrm{g}}-V_{0}\right)^{3} .
$$

For $x_{\min }<-2 \mathrm{~mm}$, integration according to (2) yields

$$
N_{\mathrm{s}}=A\left(V_{\mathrm{g}}-V_{0}\right)^{2}+B\left(V_{\mathrm{g}}-V_{0}\right)+C .
$$

Here $A, B$, and $C$ are constants that depend on $b, c$ and $k$. For brevity, we do not write the full expressions for $A, B$ and $C$ here. Thus for a source which is fille up to electron 'spill-over', the total number of electrons is expected to vary with the gate voltage in a nonlinear way, as given by (5) and (6). We have fitte our data to this model, and the dashed lines in Figs. 6 and 7 show the result. The agreement can be considered as quite satisfactory. The only unknown parameter in these fit is the constant $k$, which, to avoid confusion with the value calculated using the simulation, we denote as $k_{\exp }$. The value of $k_{\exp }$ obtained from these fit is $k_{\exp }=0.021$ for Fig. 6 and $k_{\exp }=0.018$ for Fig. 7. These values are in the same range as the value of $k$ obtained from the simulation. Some deviation is to be expected, because of several experimental uncertainties, such as the exact thickness of the helium film and the role of immobile electrons, trapped at irregularities on the substrate, as found in earlier experiments [16]. Offsets in potential due to the difference in the work functions of gold and silicon may also play a role, although we fin no evidence of this effect in our measurements. The fit give the value of the gate voltage at which $x_{\min }=-2 \mathrm{~mm}$ as $V_{\mathrm{g}}=5.3 \mathrm{~V}$. This value marks the boundary between fittin with (5) and (6).

From the results shown in Figs. 6 and 7 one can determine the electron density at the entrance of the gate channel, $n(0)$, using the model outlined above. The results, presented in Fig. 9, show that the maximum values of $n(0)$ reach $8 \times 10^{9} \mathrm{~cm}^{-2}$ and $1 \times 10^{10} \mathrm{~cm}^{-2}$ for Figs. 6 and 7 respectively. Note that these densities are distinctly higher than the maximum average densities $N_{S} / A_{\text {source }}$ (where $A_{\text {source }}$ is the source area) for Figs. 6 and 7, which are on the order of $4.1 \times 10^{9} \mathrm{~cm}^{-2}$ and $4.3 \times 10^{9} \mathrm{~cm}^{-2}$.

\section{Conclusions}

In the experiments presented here we have shown that it is possible to store surface state electrons in the source area of a FET structure up to a density where electron 

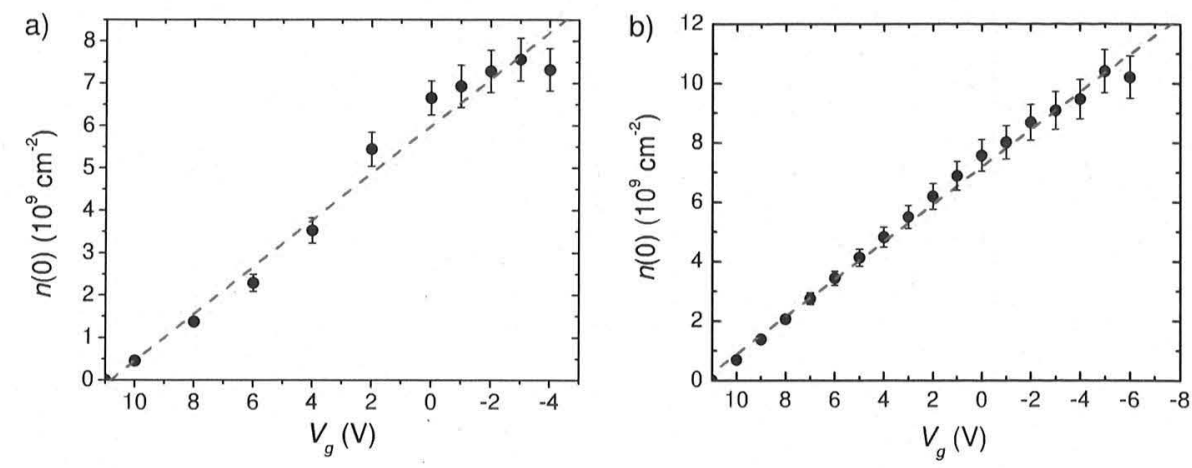

Fig. 9 (Color online) Electron density $n(0)$ in front of the gate channel as a function of the gate voltage, for the situation that the reservoir $\mathrm{S}$ is fille up to electron spill-over at the respective value of $V_{\mathrm{g}}$. (a) For the data set of Fig. 6; (b) for the data set of Fig. 7. The dashed lines are linear fit to the data

spill-over across the potential barrier of the gate sets in. The storage capacity of the source is found to be a nonlinear function of the gate voltage. This can be ascribed to the inhomogeneous spatial distribution of the electron density, which - due to the potential distribution in the silicon substrate-has a maximum at the entrance of the gate channel and decreases linearly with distance from the gate. The electron density in front of the gate channel can be tuned by the potential gradient in the substrate and the gate voltage, which is important for further transport measurements in this system. The maximum densities investigated here have been kept below $10^{10} \mathrm{~cm}^{-2}$, in order to avoid complications resulting from a breakthrough of charges through the helium film (For higher electron densities a pronounced thinning of the helium fil takes place, due to the electrostatic pressure of the SSE [17], which increases the probability for electron breakthrough.) In the density range investigated here, the system of $2 \mathrm{D}$ electrons is either in the flui or in the Wigner crystal phase $[18,19]$. Investigations at higher densities, as they are relevant for the degenerate Fermi regime, will be the subject of a separate paper, as well as studies of the electron transport through the channel of the FET structure. However, the results presented here are essential for the interpretation of all the experiments carried out with surface state electrons in FET structures.

Acknowledgements We would like to thank, DAAD (German Academic exchange Service) for supporting this work, Valeri Shikin for discussions, Louis Kukk for helping to improve some technical aspects of the experimental set-up, and Richard Rau for helping in the analysis of the data.

\section{References}

1. E. Andrei, Two-Dimensional Electron Systems on Helium and Other Cryogenic Substrates (Kluwer Academic, Dordrecht, 1997)

2. Y.P. Monarkha, K. Kono, Two-Dimensional Coulomb Liquids and Solids (Springer, Berlin, 2004)

3. Y.P. Monarkha, S. Ito, K. Shirahama, K. Kono, Phys. Rev. Lett. 78, 2445 (1997)

4. C.C. Grimes, G. Adams, Phys. Rev. Lett. 42, 795 (1979)

5. D.G. Rees, I. Kuroda, C.A. Marrache-Kikuchi, M. Höfer, P. Leiderer, K. Kono, Phys. Rev. Lett. 106, $026803(2011)$ 
6. H. Ikegami, H. Akimoto, K. Kono, Phys. Rev. B 82, 201104(R) (2010)

7. P.M. Platzman, M.I. Dykman, Science 284, 5422 (1999)

8. D.I. Schuster, A. Fragner, M.I. Dykman, S.A. Lyon, R.J. Schoelkopf, Phys. Rev. Lett. 105, 40503 (2010)

9. P. Leiderer, Physica B 126, $92(1984)$

10. T. Guenzler, B. Bitnar, G. Mistura, S. Neser, P. Leiderer, Science 361/362, 831 (1996)

11. B.J. van Wees et al., Phys. Rev. Lett. 60, 848 (1988)

12. D.A. Wharam et al., J. Phys. C, Solid State Phys. 21, L209 (1988)

13. Y.B. Levinson, M.I. Lubin, E.V. Sukhorukov, Phys. Rev. B 45, 11936 (1992)

14. J. Klier, I. Doicescu, P. Leiderer, J. Low Temp. Phys. 121, 603 (2000)

15. J. Wilks, The Properties of Liquid and Solid Helium (Clarendon, Oxford, 1967)

16. J. Klier, T. Guenzler, A. Wuerl, P. Leiderer, G. Mistura, E. Teske, P. Wyder, V. Shikin, J. Low Temp. Phys. 122, 451 (2001)

17. H. Etz, W. Gombert, W. Idstein, P. Leiderer, Phys. Rev. Lett. 53, 2567 (1984)

18. R.S. Crandall, R. Williams, Phys. Lett. A 34, 404 (1971)

19. F.M. Peeters, P.M. Platzman, Phys. Rev. Lett. 50, 2021 (1983) 